\title{
The E89K Mutation in the Matrix Protein of the Measles Virus Affects In Vitro Cell Death and Virus Replication Efficiency in Human PBMC
}

\author{
Jianbao Dong ${ }^{1,2,3}$, Wei Zhu ${ }^{1}$, Akatsuki Saito ${ }^{1}$, Yoshitaka Goto ${ }^{1}$, Hiroyuki Iwata ${ }^{4}$, Takeshi Haga ${ }^{*}, 1$ \\ ${ }^{I}$ Department of Veterinary Microbiology, University of Miyazaki, Miyazaki 889-2192, Japan \\ ${ }^{2}$ Research Fellow DC of the Japan Society for the Promotion of Science, Yamaguchi University, Yamaguchi 753-8511, Japan \\ ${ }^{3}$ The United Graduate School of Veterinary Science, Yamaguchi University, Yamaguchi 753-8511, Japan \\ ${ }^{4}$ Department of Veterinary Medicine, Yamaguchi University, Yamaguchi 753-8511, Japan
}

\begin{abstract}
Matrix protein is known to have an important role in the process of virus assembly and virion release during measles virus replication. In the present in vitro study, a single mutation of $\mathrm{E} 89 \mathrm{~K}$ in the matrix protein was shown to affect cell death and virus replication efficiency in human PBMC. One strain with this mutation caused less cell death than the parental virus, and possessed high virus replication efficiency. Moreover, by Annexin V-FITC staining, polycaspase FLICA staining, and double labeling with poly-caspase FLICA and the Hoechst stain, the cell death seen was shown to be apoptosis.
\end{abstract}

Keywords: Measles virus, human PBMC, E89K mutation, cell death, replication efficiency.

\section{INTRODUCTION}

Measles, which is caused by the measles virus $(\mathrm{MeV})$, remains a major contributor to childhood morbidity and mortality, particularly in developing countries with low vaccine coverage rates. $\mathrm{MeV}$ is an enveloped virus belonging to the genus Morbillivirus of the family Paramyxoviridae having a nonsegmental negative-strand RNA genome of 15,894 nucleotides in length. The $\mathrm{MeV}$ genome contains six sequential genes that code for structural proteins: nucleocapsid $(\mathrm{N})$, phospho- $(\mathrm{P})$, matrix $(\mathrm{M})$, fusion $(\mathrm{F})$, hemagglutinin $(\mathrm{H})$, and large $(\mathrm{L})$ proteins $\left(5^{\prime}-\mathrm{N}-\mathrm{P}-\mathrm{M}-\mathrm{F}-\mathrm{H}-\mathrm{L}-\right.$ 3 '). In addition, the $\mathrm{MeV}$ virion contains two nonstructural proteins, $\mathrm{V}$ and $\mathrm{C}$. The $\mathrm{V}$ protein is generated from an edited mRNA that contains an extra $\mathrm{G}$ residue, while the $\mathrm{C}$ protein is alternatively translated from a downstream start signal [1].

It is well known that the $\mathrm{M}$ protein of $\mathrm{MeV}$ and other members of the Paramyxoviridae family plays an important role in the process of virus assembly and virion release, which takes place at the plasma membrane [2-5]. In addition, many other functions have been discovered and confirmed. For example, $\mathrm{M}$ protein can inhibit polymerase activity and viral transcription during virus replication $[6,7]$. It also can affect efficient growth and cell-cell fusion [8,9]. A previous study revealed that the E89K substitution mutation in $\mathrm{M}$ protein plays a key role in wild-type $\mathrm{MeV}$ adaptation in the cotton rat and cotton rat lung (CRL) cells [10].

Human peripheral blood mononuclear cells (PBMC) are the main target cells of natural $\mathrm{MeV}$ infection. In order to determine how the E89K-mutated $\mathrm{M}$ protein affects the

*Address correspondence to this author at the Department of Veterinary Microbiology, University of Miyazaki, 1-1 Gakuen Kibanadai Nishi, Miyazaki 889-2192, Japan; Tel: +81-985-58-7575; Fax: +81-985-58-7575; E-mail: a0d518u@cc.miyazaki-u.ac.jp phenotype of PBMC and virus, cell death and virus replication efficiency were analyzed in this in vitro study. Results indicate that the mutant virus causes less cell death than the parental virus, and possesses high replication efficiency in human PBMC.

\section{MATERIALS AND METHODS}

\section{Cell Culture and Virus Infection}

Adapted virus MV99YC7, which adapted well to CRL cells after serial passages, possesses a mutation in the $\mathrm{M}$ gene causing E89K substitution and one silent mutation in the L gene, as compared to the parental virus MV99Y [10]. Human PBMC were isolated from healthy adults using Ficoll-Paque PREMIUM (GE Healthcare Bio-science, Piscataway, NJ). Purified PBMC were stimulated with 5 $\mu \mathrm{g} / \mathrm{ml}$ concanavalin A (ConA) and cultured in $10 \%$ FCSRPMI medium [RPMI 1640 with $2 \mathrm{mM}$ L-glutamine and sodium bicarbonate (Sigma, St Louis, MO) containing 10\% heat-inactivated fetal calf serum (FCS) (JRH Bioscience, Overland Park, Kansas)] for 24 hours at $37{ }^{\circ} \mathrm{C}$ in $5 \% \mathrm{CO}_{2}$. Non-adherent cells were harvested, washed three times with PBS and then infected with MV99Y or MV99YC7 at an MOI of 1.0 by incubation at $37{ }^{\circ} \mathrm{C}$ in $5 \% \mathrm{CO}_{2}$ for 1 hour. Mock-infected PBMC served as negative controls.

\section{Cell Labeling, Staining and Analysis}

After infection, cells were washed three times with $37^{\circ} \mathrm{C}$ RPMI 1640 to remove unattached virus and then cultured in $10 \%$ FCS-RPMI medium containing $5 \mu \mathrm{g} / \mathrm{ml}$ ConA; 100 $\mu \mathrm{g} / \mathrm{ml}$ Virus Replication Inhibiting Peptide (Z- D- Phe- PheGly; Peptide Institute Inc. Osaka, Japan) [11] was added to the medium to block a second round of infection by progeny viruses. Cells were harvested 24, 48, 72, and 96 hours postinfection (hpi), and then were stained with Annexin V-FITC (MBL, Nagoya, Japan) and poly-caspases FLICA (Fluorochrome Inhibitor of Caspases; Immunochemistry 
Technologies, Bloomington, $\mathrm{MN}$ ), respectively. The fluorescence was measured using FACSCanto II (BD Biosciences, San Jose, CA) according to the manufacturer's instruction. Double labeling with poly-caspases FLICA and Hoechst stain was performed for cells harvested at different time points and observed under fluorescence microscope.

\section{Assessment of Comparable Virus Input}

In parallel experiments, viruses were harvested and titrated with B95a cells, the Epstein-Barr virus-transformed cotton-top tamarin B cell line [12]. To determine whether an equal amount of input virus (MV99Y and MV99YC7 strains) had infected the PBMC, quantitative RT-PCR assays were performed using one pair of specific primers, based on the nucleotide sequences of the $\mathrm{M}$ gene: RtmF (5'TACAACAACACCCCACTAAC-3') and RtmR (5'AAACAACACGGAACCTCTGC-3'). After undergoing the infection procedure described above, $5 \times 10^{5}$ infected PBMC cells were harvested and RNA was extracted using the RNeasy Mini Kit (Qiagen, Hilden, Germany). Viral RNA was quantified in six repeats of real-time RT-PCR using One Step SYBR PrimeScript PLUS RT-PCR Kit (Takara, Shiga, Japan).

\section{Apoptosis Assessment}

In order to determine whether viral replication contributes to apoptosis induction, UV-inactivated MV99Y and MV99YC7 were generated by ultraviolet (UV) irradiation at $2 \mathrm{~J} / \mathrm{cm}^{2}$ as described in a previous report [13]. PBMC were infected, as described above, with these at an MOI of 1.0, harvested at 24, 48, 72, and $96 \mathrm{hpi}$, stained with Annexin V-FITC and poly-caspase FLICA, and then analyzed by flow cytometry.

\section{Statistical Analysis}

Data were analyzed using Excel software (Microsoft). Variance analysis was performed with single factor (One Way) ANOVA tests.

\section{RESULTS}

MeV-infected PBMC cell death was detected with Annexin V staining and flow cytometric analysis. The mutant MV99YC7 strain caused significantly $(\mathrm{P}<0.01)$ less cell death than the parental strain MV99Y at all time points studied (Fig. 1). Compared to uninfected control cells, MV99Y-infected cells were not different at $24 \mathrm{hpi}$, but a highly significant difference $(\mathrm{P}<0.01)$ emerged between 48 and 96 hpi.

To determine whether cell death was caused by viral replication, $\mathrm{UV}$-inactivated $\mathrm{MeV}$ infection was carried out; no significant differences were found among UV-inactivated MV99Y-infected, UV-inactivated MV99YC7-infected cells and uninfected PBMC (Fig. 1), suggesting that viral replication contributes to the cell death.
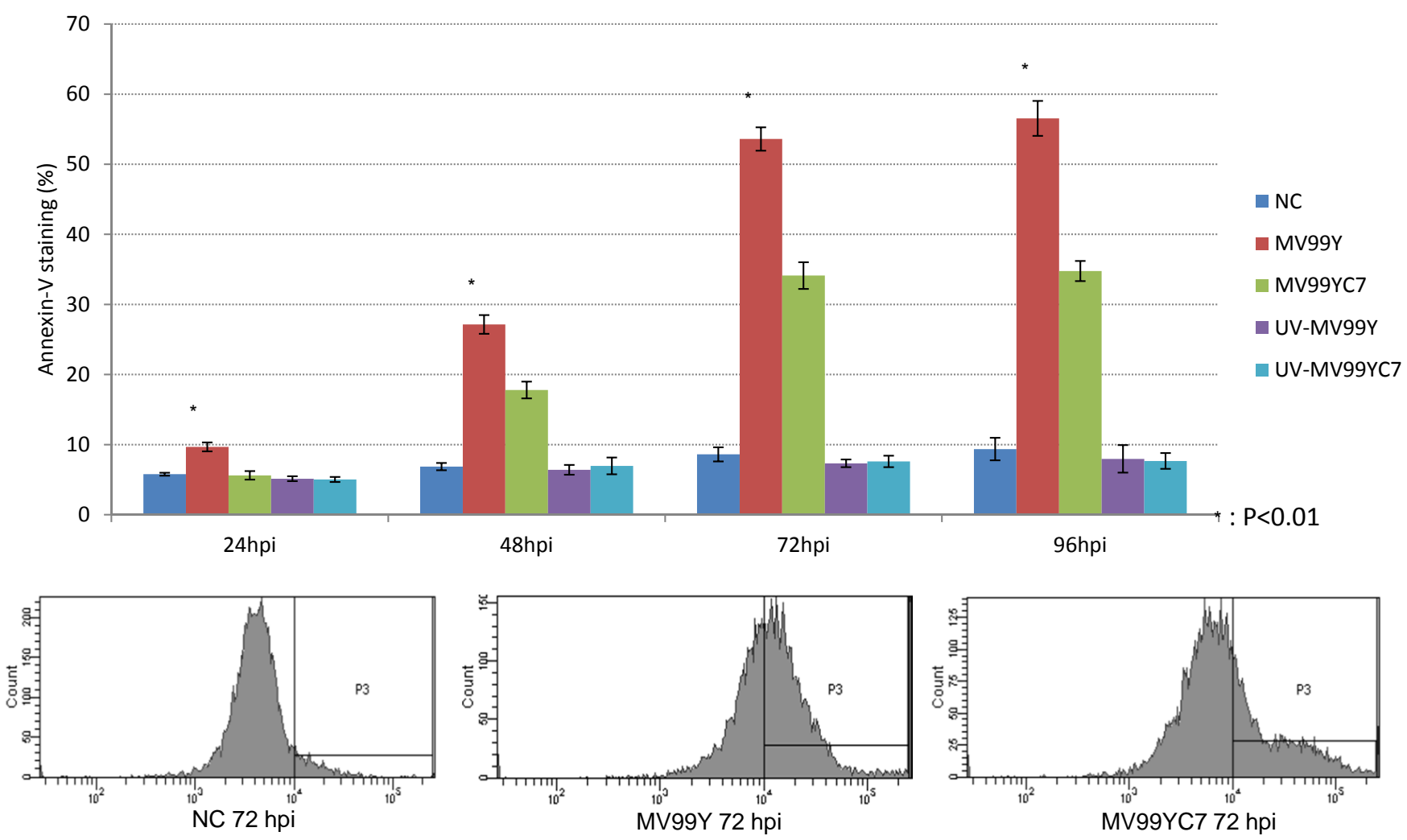

Fig. (1). Flow cytometric analysis of cell death by staining with Annexin V-FITC. Human PBMC were infected with MV99Y, MV99YC7, UV-inactivated MV99Y or UV-inactivated MV99YC7 at an MOI of 1.0 and harvested at 24, 48, 72 and 96 hours post-infection (hpi). Flow cytometry using FACSCanto II (BD) with a single laser emitting excitation light at $488 \mathrm{~nm}$. Percent Annexin V positive cells determined with a gate draw from an unstained control. Lower panel: $\mathrm{X}$ - and $\mathrm{Y}$-axes; cell number and fluorescence intensity, respectively. P3: gate for positive cells. NC: negative control. UV-MV99Y and UV-MV99YC7: PBMC infected with UV-inactivated virus strains. Mean (SD); $\mathrm{n}=6$. *Significant difference between MV99Y and MV99YC7 $(\mathrm{P}<0.01)$. 


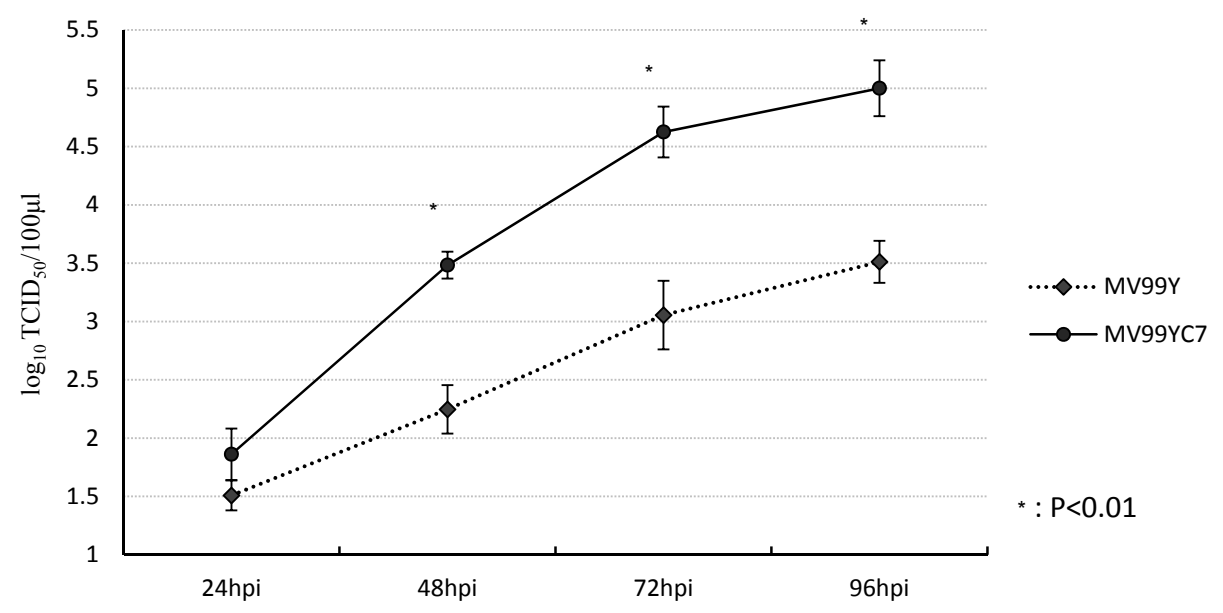

Fig. (2). Replication kinetics of viruses in PBMC. Human PBMC were infected with MV99Y or MV99YC7 at an MOI of 1.0. Supernatants harvested at 24, 48, 72, and 96 hours post-infection (hpi). TCID $_{50}$ of cell-free viruses determined using B95a cells; virus titer expressed as $\log _{10} \mathrm{TCID}_{50} / 100 \mu \mathrm{l}$ supernatant. NC: negative control. Mean $(\mathrm{SD}) ;(\mathrm{n}=6)$. *Significant difference between MV99Y and MV99YC7 $(\mathrm{P}<0.01)$.

Virus replication kinetics are shown in Fig. (2); the MV99YC7 strain possessed higher replication efficiency than MV99Y at 48, 72 and 96 hpi $(\mathrm{P}<0.01)$. Quantitative real-time RT-PCR assays were performed out to assess the virus particle input, including receptor-bound virus. No significant differences were found between MV99Y- and MV99YC7-infected cells. Considered together, these results indicated that the mutant possessed higher growth efficiency than the parental strain in this study.

Cell death has been divided into two main types: apoptosis and necrosis. In apoptosis, caspases are well known to participate in a series of reactions triggered in response to proapoptotic signals, resulting in cleavage of protein substrates, causing cell disassembly [14]. In order to identify the type of cell death caused by $\mathrm{MeV}$ in this study, double labeling with poly-caspase FLICA and the Hoechst stain (which reacts with the condensed nuclei of dying cells) were carried out. Only a few positive cells were seen in the mock-infected PBMC. In contrast, PBMC infected with MV99Y and MV99YC7 were definitely positive for both labels (Fig. 3), indicating that the cell death seen in the present study was due to apoptosis.

Single staining with poly-caspase FLICA was also performed. Flow cytometric analysis revealed a low percentage (3.9 to $6.9 \%$ ) of poly-caspase positive mockinfected cells at all time points. At $24 \mathrm{hpi}$, a low percentage of MV99Y- and MV99YC7-infected cells was detected $(7.85 \%$ and $6.8 \%$, respectively; $\mathrm{P}>0.05)$; both were significantly different $(\mathrm{P}<0.01)$ than mock-infected cells (3.9\%). Between 48 and $96 \mathrm{hpi}$, significant differences within the MV99Y- and MV99YC7-infected cultures emerged; infected cell numbers increased from $36.4 \%$ to $56.7 \%$ and from $16.3 \%$ to $37.8 \%$, respectively. In addition, during that time period differences between parental and mutant infections were significant $(\mathrm{P}<0.01)$ (Fig. 4).
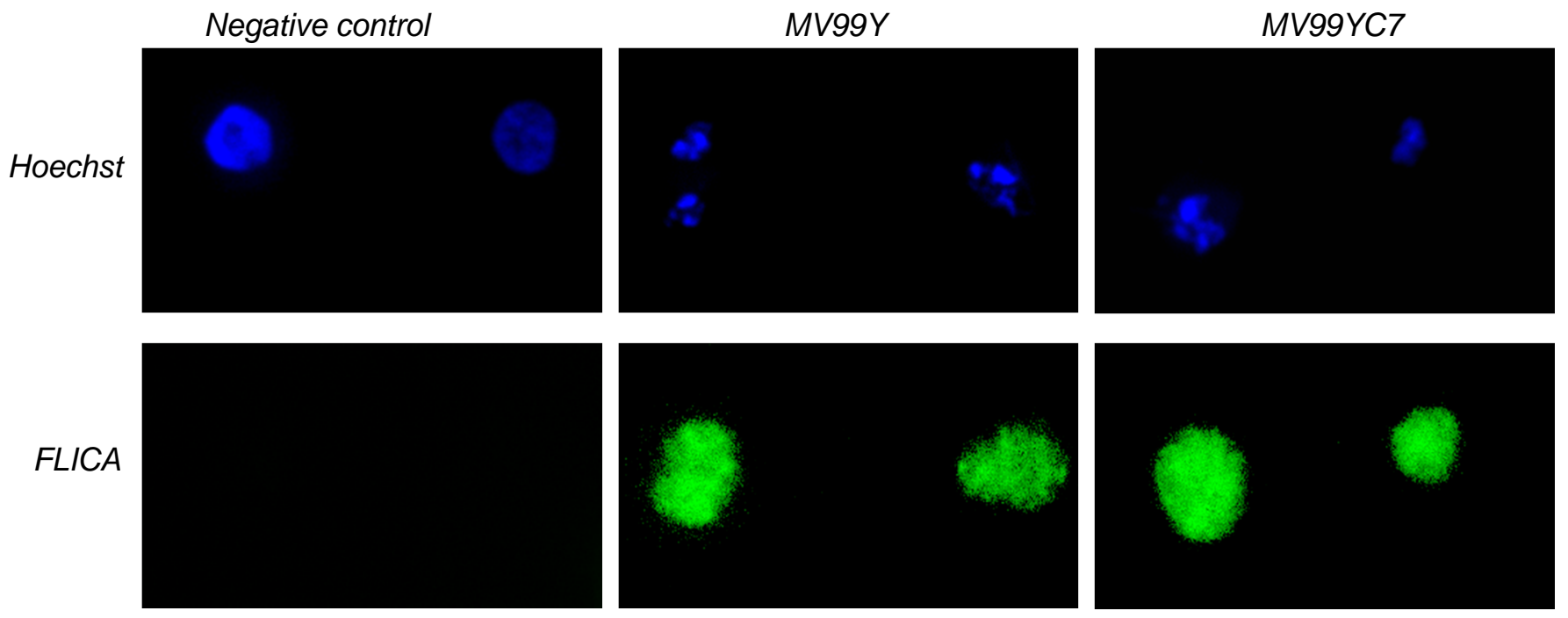

Fig. (3). Double labeling with poly-caspase FLICA and Hoechst staining. Human PBMC infected with MV99Y or MV99YC7 at an MOI of 1.0. Cells harvested at 72 hours post-infection (hpi). Caspase activity (green) detected using a band pass filter (excitation $488 \mathrm{~nm}$, emission $520 \mathrm{~nm}$ ). Nuclear staining by Hoechst stain (blue) revealed using a UV-filter (excitation $365 \mathrm{~nm}$, emission $480 \mathrm{~nm}$ ). 

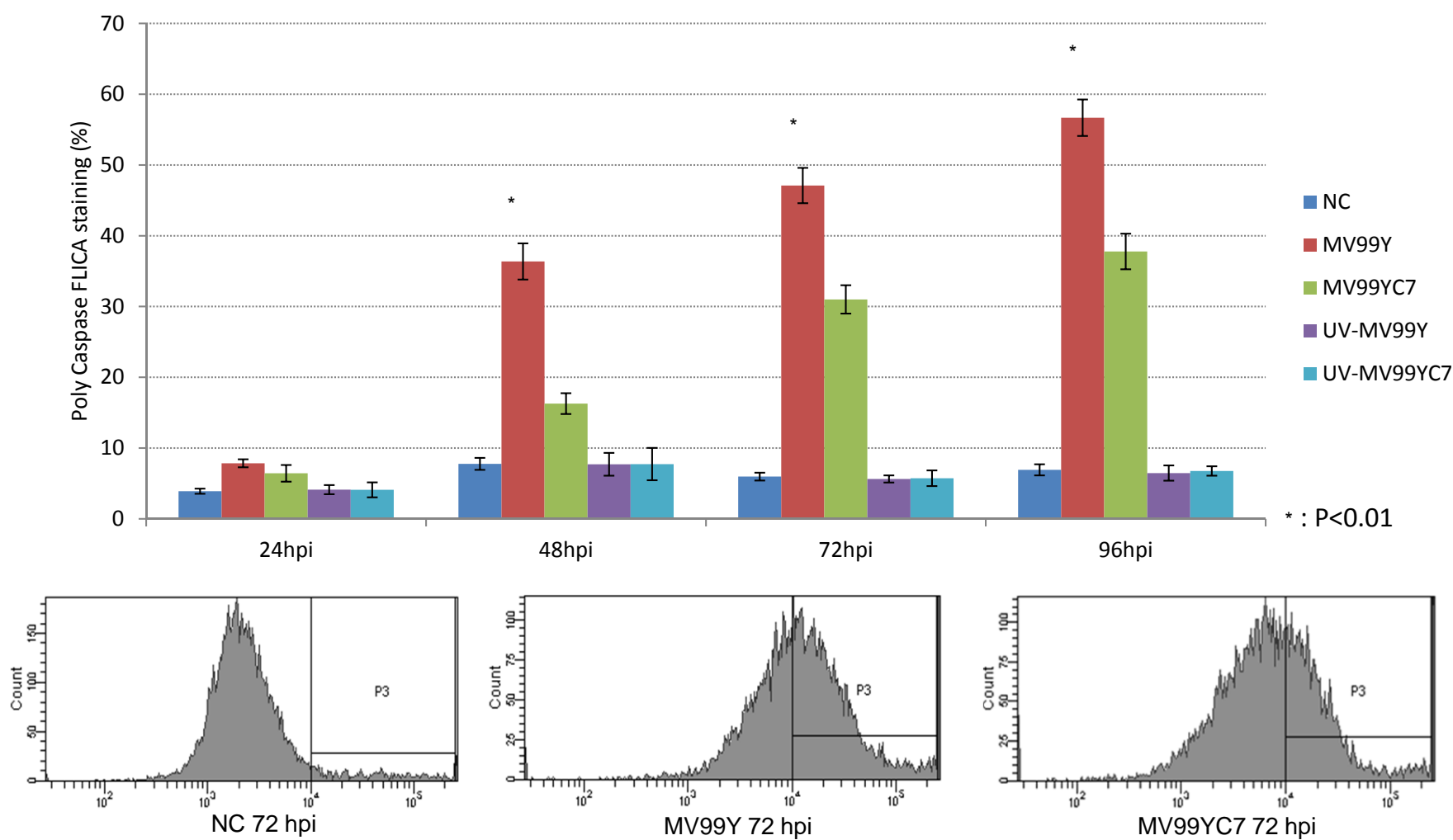

Fig. (4). Flow cytometric analysis of apoptotic cells by staining with poly-caspase FLICA. Human PBMC infected with MV99Y, MV99YC7, UV-inactivated MV99Y or UV-inactivated MV99YC7 at an MOI of 1.0. Cells harvested at 24, 48, 72 and 96 hours postinfection (hpi). Flow cytometry by FACSCanto II (BD) using a single laser emitting excitation light at $488 \mathrm{~nm}$. Percentage of poly-caspaseFLICA-positive cells determined with a gate draw from an unstained control. Lower panel: X- and Y-axes; cell number and fluorescence intensity, respectively. P3: gate for positive cells. NC: negative control. UV-MV99Y and UV-MV99YC7: PBMC infected with UVinactivated virus strains. Mean (SD); $\mathrm{n}=6$. * Significant difference between MV99Y and MV99YC7 (P<0.01).

UV-inactivated MV99Y and MV99YC7 were utilized to determine whether viral replication contributes to apoptosis induction. A low percentage of caspase-positive cells was detected in UV-inactivated parental- and mutant-infected cells at all time points (Fig. 4), and there was no significant difference among them and mock-infected cells. This indicates indirectly that viral replication contributed to apoptosis in the present study.

\section{DISCUSSION}

It is known that $\mathrm{MeV}$ infection can cause lymphopenia and degradation of cell-mediated immunity, and that virulent strains usually cause more severe immunosuppression than strains used in vaccines. However, the precise mechanisms involved remain unclear, although motifs of $\mathrm{MeV}$ proteins might be pertinent. Compared with the wild-type Edmoston strain, all vaccine strains (AIK-C, Moraten, Schwarz, Rubeovax, Zagreb, CAM-70, Changchun-47, Leningrad-4 and Shanghai-191) possess the $\mathrm{E} 89 \mathrm{~K}$ mutant $\mathrm{M}$ protein $[15$, 16]. It has remained unclear whether this mutation is associated with $\mathrm{MeV}$ virulence. Results of the present study indicate that the mutant virus caused less cell death than the parent. Since only the infection on monkey model can show attenuation of $\mathrm{MeV}$ strain. Therefore, further investigation is necessary to clarify the relationship between virulence and the E89K mutation.

P64S and E89K M protein substitutions were reported to be responsible for efficient virus growth in Vero cells [9].
However, recombinant viruses with these mutations had a growth disadvantage in CD150-positive B95a cells [9]. Moreover, interactions of the $\mathrm{M}$ protein with the cytoplasmic tails of the $\mathrm{H}$ protein modulated $\mathrm{MeV}$ growth in different cell types [8]. In this study, we presented E89K substitution in the $M$ protein contributed to virus replication efficiency in human PMBC.

The E89K mutation in the M protein has been shown to play a key role in wild-type $\mathrm{MeV}$ adaptation in cotton rat and CRL cells [10]. This may be explained by the possibility that the mutation facilitates a strong interaction between the $\mathrm{M}$ protein and the cytoplasmic tail of the $\mathrm{H}$ protein, thus enhancing assembly of virus particles [8,9]. Interestingly, the result of the present study provide another presumed mechanism that the E89K mutation inhibited the cell apoptosis to aid virus adaption. Further studies are required to elucidate the precise mechanisms involved.

In conclusion, compared with the parental strain MV99Y, the MV99YC7 mutant strain caused less cell death and possessed higher replication efficiency in human PBMC in vitro. Furthermore, the cell death seen was shown to be due to apoptosis.

\section{ACKNOWLEDGEMENTS}

This work was partly supported by the Project for Zoonoses Education and Research, University of Miyazaki, and by a grant from the Japan Society for the Promotion of Science (No. KAKENHI22780272). 


\section{CONFLICT OF INTEREST}

Declared none.

\section{REFERENCES}

[1] Knipe DM, Howley PM. Fields Virology. 5th ed. Philadelphia: Lippincott Williams \& Wilkins 2007.

[2] Cathomen T, Naim HY, Cattaneo R. Measles viruses with altered envelope protein cytoplasmic tails gain cell fusion competence. J Virol 1998; 72: 1224-34.

[3] Garoff H, Hewson R, Opstelten DJ. Virus maturation by budding. Microbiol Mol Biol Rev 1998; 62: 1171-90.

[4] Schmitt AP, He B, Lamb RA. Involvement of the cytoplasmic domain of the hemagglutinin-neuraminidase protein in assembly of the paramyxovirus simian virus 5. J Virol 1999; 73: 8703-12.

[5] Takimoto T, Portner A. Molecular mechanism of paramyxovirus budding. Virus Res 2004; 106: 133-45.

[6] Reuter T, Weissbrich B, Schneider-Schaulies S, et al. RNA interference with measles virus $\mathrm{N}, \mathrm{P}$, and L mRNAs efficiently prevents and with matrix protein mRNA enhances viral transcription. J Virol 2006; 80: 5951-7.

[7] Suryanarayana K, Baczko K, ter Meulen V, et al. Transcription inhibition and other properties of matrix proteins expressed by $\mathrm{M}$ genes cloned from measles viruses and diseased human brain tissue. J Virol 1994; 68: 1532-43.

[8] Tahara M, Takeda M, Yanagi Y. Altered interaction of the matrix protein with the cytoplasmic tail of hemagglutinin modulates measles virus growth by affecting virus assembly and cell-cell fusion. J Virol 2007; 81: 6827-36.

[9] Tahara M, Takeda M, Yanagi Y. Contributions of matrix and large protein genes of the measles virus edmonston strain to growth in cultured cells as revealed by recombinant viruses. J Virol 2005; 79: 15218-25.

[10] Dong JB, Saito A, Mine Y, et al. Adaptation of wild-type measles virus to cotton rat lung cells: E89K mutation in matrix protein contributes to its fitness. Virus Genes 2009; 39: 330-4.

[11] Richardson CD, Scheid A, Choppin PW. Specific inhibition of paramyxovirus and myxovirus replication by oligopeptides with amino acid sequences similar to those at the N-termini of the F1 or HA2 viral polypeptides. Virology 1980; 105: 205-22.

[12] Kobune F, Sakata H, Sugiura A. Marmoset lymphoblastoid cells as a sensitive host for isolation of measles virus. J Virol 1990; 64: 700-5.

[13] Haga T, Murayama N, Shimizu Y, et al. Analysis of antibody response by temperature-sensitive measles vaccine strain in the cotton rat model. Comp Immunol Microbiol Infect Dis 2009; 32: 395-406.

[14] Slee EA, Adrain C, Martin SJ. Serial killers: ordering caspase activation events in apoptosis. Cell Death Differ 1999; 6: 1067-74.

[15] Bankamp B, Takeda M, Zhang Y, et al. Genetic characterization of measles vaccine strains. J Infect Dis 2011; 204(Suppl 1): S533-48.

[16] Parks CL, Lerch RA, Walpita P, et al. Comparison of predicted amino acid sequences of measles virus strains in the Edmonston vaccine lineage. J Virol 2001; 75: 910-20.

(C) Dong et al.; Licensee Bentham Open.

This is an open access article licensed under the terms of the Creative Commons Attribution Non-Commercial License (http: //creativecommons.org/licenses/by$\mathrm{nc} / 3.0 /$ ) which permits unrestricted, non-commercial use, distribution and reproduction in any medium, provided the work is properly cited. 